\title{
MGMT promoter methylation as a potential prognostic marker for acute leukemia
}

\author{
Dominika Sobieszkoda ${ }^{1}$, Joanna Czech ${ }^{1,2}$, Natalia Gablo ${ }^{1,2}$, Marta Kopanska ${ }^{1,2}$, \\ Jacek Tabarkiewicz ${ }^{1,3}$, Agnieszka Kolacinska ${ }^{4,5}$, Tadeusz Robak ${ }^{6}$, Izabela Zawlik ${ }^{1,2}$
}

\author{
${ }^{1}$ Centre for Innovative Research in Medical and Natural Sciences, Faculty of Medicine \\ University of Rzeszow, Rzeszow, Poland \\ 2Department of Genetics, Chair of Molecular Medicine, Faculty of Medicine, \\ University of Rzeszow, Rzeszow, Poland \\ ${ }^{3}$ Department of Immunology, Chair of Molecular Medicine, Faculty of Medicine, \\ University of Rzeszow, Rzeszow, Poland \\ ${ }^{4}$ Department of Head and Neck Cancer Surgery, Medical University of Lodz, Lodz, \\ Poland \\ ${ }^{5}$ Department of Surgical Oncology, Cancer Center, Copernicus Memorial Hospital, \\ Lodz, Poland \\ ${ }^{6}$ Department of Hematology, Medical University of Lodz, Copernicus Memorial \\ Hospital, Lodz, Poland
}

Submitted: 7 September 2016

Accepted: 14 November 2016

Arch Med Sci 2017; 13, 6: 1433-1441

DOI: https://doi.org/10.5114/aoms.2017.71067

Copyright @ 2017 Termedia \& Banach

\section{Abstract}

Introduction: It has been proved that genetic and epigenetic changes play a significant role in the development and progression of acute leukemia. The aim of our study was to evaluate the frequency and prognostic implications of genetic and epigenetic alterations in p15, MGMT, DNMT3A and TP53 genes in acute leukemias.

Material and methods: We included in the study 59 patients with acute leukemia. Evaluation of TP53 and DNMT3A mutations was performed using sequencing analysis and PCR-RFLP, respectively. Methylation status of MGMT and $p 15$ genes was evaluated using MSP and COBRA, respectively. For assessment of global DNA methylation ELISA-based kit was used.

Results: We found that overall survival was higher for ALL patients. MGMT promoter methylation was significantly associated with patients age at the time of diagnosis $(p=0.03)$. TP53 and DNMT3A mutations were observed only in AML patients $(16.67 \%$ and $8.8 \%$, respectively). Patients with acute leukemia and $p 15$ promoter methylation had significantly more frequently mutated TP53 gene $(p=0.04)$ and AML patients with $p 15$ promoter methylation had significantly more frequently detected global hypomethylation of DNA $(p=0.009)$. In the group of ALL patients we noted an opposite trend: only patients negative for $p 15$ promoter methylation were characterized by global DNA hypomethylation.

Conclusions: Our findings demonstrate that MGMT promoter methylation can have a considerable impact on the development of acute leukemia in older patients. DNMT3A and TP53 mutations may play a significant role in AML development. However, further studies conducted in a larger cohort of patients are needed to determine its clinical utility.

Key words: acute leukemia, MGMT, DNMT3A, p15, TP53.
Corresponding author: Assoc. Prof. Izabela Zawlik Center for

Innovative Research

in Medical and

Natural Sciences

Faculty of Medicine

University of Rzeszow

1 a Warzywna St

35-959 Rzeszow, Poland

Department of Genetics

Chair of Molecular

Medicine

Faculty of Medicine

University of Rzeszow

2 a Kopisto St

35-959 Rzeszow, Poland

Phone: +48178516810

E-mail: izazawlik@yahoo.com 


\section{Introduction}

Leukemias are a clinically and genetically heterogeneous group of diseases that begin in early blood-forming cells found in the bone marrow. It is a result of genetic mutations and transformation of a single early progenitor lymphoid or myeloid cell. Acute lymphoblastic leukemia (ALL) is the most common leukemia in children, accounting for up to $80 \%$, and only for $20 \%$ of leukemias in adults [1], whereas acute myeloblastic leukemia $(\mathrm{AML})$ is more frequent in patients at an older age where the median age at the time of diagnosis is about 70 years [2].

Genetics plays an increasingly important role in the classification, risk stratification, and management of acute leukemias [3]. For the past several years, an increasing number of gene mutations deregulating expression of these genes and epigenetic changes have been established in the pathogenesis of acute leukemias $[4,5]$. The most commonly mutated gene in people who have cancer is the TP53 tumor-suppressor gene [6]. The P53 protein, widely known as a transcription factor, plays an important role in the maintenance of genetic stability and preventing cancer formation. Loss of P53 function is associated with various characteristics of tumors, including genetic instability or deregulation of the cell cycle [7]. It has been reported that these mutations are associated with a poor prognosis and treatment failure in both ALL and AML $[8,9]$. It has also been shown that DNMT3A mutations are essential for early development of acute leukemia [10, 11]. DNMT3A(DNA(cytosine-5-)-methyltransferase $3 \alpha$ ), a member of the de novo methyltransferases, is closely associated with epigenetic modifications in mammalian development and diseases. More recent studies have identified that most of the recurrent somatic mutations in DNMT3A in AML are heterozygous [10]. The normal function of DNMT3A can be disturbed by these mutations, which subsequently results in an abnormality of epigenetic modification. In approximately $20 \%$ of AML cases mutations in the DNMT3A gene have been observed and correlated with a poor clinical outcome [12, 13].

There is more and more evidence that epigenetic changes play an important role in the development of cancer, including acute leukemia. DNA methylation abnormalities are common in a variety of cancers as well as in development. Global hypomethylation and hypermethylation, which repress transcription of the promoter regions of tumor suppressor genes leading to gene silencing, have been recognized as a cause of oncogenesis [14]. Numerous changes in the methylation of promoter regions of various genes have been observed in patients with acute leukemia $[15,16]$.
The $p 15$ gene, inhibitor of cyclin-dependent kinases 4/6 (CDK4/6), is among the most frequent targets of aberrant methylation in acute leukemias [17]. Hypermethylation of the DNA repair gene MGMT $\left(\mathrm{O}^{6}\right.$-methylguanine DNA methyltransferase) has been found in $A M L$, but its potential prognostic value is not yet fully elucidated $[18,19]$.

The aim of the study was to determine whether the MGMT and p15 promoter hypermethylation and global DNA hypomethylation, as well as DNMT3A and TP53 mutations, have an impact on the development acute leukemias. Moreover, we wanted to investigate the relationship between examined molecular parameters and time at diagnosis and overall survival in patients with acute leukemia. A better understanding of the underlying genetic and epigenetic processes may lead to gaining insight into the mechanism of leukemogenesis in $A L L$ and $A M L$, as well as providing prognostic information and prospective healing targets.

\section{Material and methods}

\section{Materials}

Fifty-nine Caucasian patients (19 ALL: 11 male and 8 female; 36 AML: 22 male and 14 female; 4 without a precise diagnosis: 3 male and 1 female; median age: 54) involved in this study were recruited at the Department of Hematology, Medical University of Lodz, between 1998 and 2001. A detailed list of all patients' characteristics is shown in Table I. Ethics committee approval was obtained from the Institutional Review Board of the Medical University of Lodz (no. RNN/226/11/KE). Peripheral blood or bone marrow samples were obtained from the patients prior to the initiation of therapy. Genomic DNA from the peripheral blood or the bone marrow blast cells was isolated by lysis of the cells with sodium dodecyl sulfate (SDS), digestion with proteinase $\mathrm{K}$ at $37^{\circ} \mathrm{C}$ overnight followed by phenol/chloroform extraction and ethanol precipitation.

\section{Sequencing analysis}

Four genomic regions of the TP53 gene (exons 5-8) were amplified by PCR, as described previously [20]. Sequence analysis was performed by the dideoxy termination method using the SequiTherm Excel DNA Sequencing Kit (Epicentre Technologies, Madison, WI) and fluorescent-labeled primers. Sequencing primers used were as follows: 5'-CAAGCAGTCACAGCACATGA-3' (forward) and 5'-AACCAGCCCTGTCGTCTCT-3' (reverse), for exon 5; 5'-CAGGCCTCTGATTCCTCACT-3' (forward) and 5'-AGACCTCAGGCGGCTCATAG-3' (reverse), for exon 6; 5'-ATCTCCTAGGTTGGCTCTGA-3' (forward) and 5'-TGGCAAGTGGCTCCTGACCT-3' (reverse), for exon 7; 5'-CTCTTTTCCTATCCTGAGTA-3' (forward) 
and 5'-CTGCTTGCTTACCTCGCTTA-3' (reverse), for exon 8. Products of the sequencing reaction were visualized and analyzed using a LiCor automated laser fluorescence sequencer.

\section{Methylation-specific PCR}

Sodium bisulfite modification of isolated genomic DNA was performed using the CpGenome DNA kit (Chemicon International Inc. Temecula, CA) according to the manufacturer's protocol. The bisulfite-treated DNA was stored at $-80^{\circ} \mathrm{C}$ until use. CpGenome Universal Methylated DNA (Chemicon International) was used as a methylation-positive control for the methylated alleles, and DNA from peripheral blood leukocytes was used as the control for unmethylated alleles. Methylation-specific PCR (MSP) for MGMT promoter methylation was performed in a two-step approach as previously reported [21]. For each PCR, methylated and unmethylated DNA was included as positive and negative controls, and water was used as a control for the PCR reaction. PCR products were separated on $3 \%$ agarose gels containing ethidium bromide. Product size of the unmethylated DNA was $91 \mathrm{bp}$ and that of the methylated DNA was $83 \mathrm{bp}$.

\section{PCR-RFLP}

DNMT3A gene mutation (R882H; GCCGC to GCCAC) was genotyped by means of polymerase chain reaction-restriction fragment length polymorphism (PCR-RFLP). Genomic DNA was amplified in a $20 \mu \mathrm{l}$ volume containing $1 \mathrm{U}$ of GoTaqFlexi DNA polymerase (Promega, Poland), $1 \mu$ of template DNA, $4 \mu$ l of $5 X$ PCR buffer, $2.0 \mathrm{mM} \mathrm{MgCl}, 0.3 \mathrm{mM}$ dNTPs (mixture of dATP, dTTP, dCTP and dGTP), and 0.6 pmol of each primer (forward: 5'-GTGATCTGAGTGCCGGGTTG-3' and reverse: 5'-TCTCTCCATCCTCATGTTCTTG-3'). The PCR cycling conditions were: 10 min of initial denaturation at $95^{\circ} \mathrm{C}$, followed by 35 cycles of denaturation for $30 \mathrm{~s}$ at $95^{\circ} \mathrm{C}$, annealing for $30 \mathrm{~s}$ at $59^{\circ} \mathrm{C}$ and extension for $1 \mathrm{~min}$ at $72^{\circ} \mathrm{C}$, followed by $7 \mathrm{~min}$ of final extension at $72^{\circ} \mathrm{C}$. A $7 \mu \mathrm{l}$ aliquot of PCR product was then digested with $3 \mathrm{U}$ of restrictive enzyme Satl (Fnu4HI) and $2 \mu$ of $1 \mathrm{X}$ BufferG (Thermo Scientific) in a final volume of $20 \mu$ l. After incubation at $37^{\circ} \mathrm{C}$ for $16 \mathrm{~h}$ restriction fragments were separated in a $2 \%$ agarose gel stained with Midori Green DNA Stain (Nippon Genetics) and visualized under UV light. Positive samples showed 3 bands (289 bp, 190 bp, 114 bp) because of the loss of a restriction site of Satl caused by the mutation.

\section{Combined bisulfite restriction analysis (COBRA)}

For methylation analysis of the $p 15$ gene we used combined bisulfite restriction analysis
Table I. Patient characteristics

\begin{tabular}{|lc|}
\hline Parameter & Value \\
\hline Age, median (range) [years] & $54(32.5-63.5)$ \\
\hline Sex: & $36(61.0 \%)$ \\
\hline Male & $23(39.0 \%)$ \\
\hline Type of leukemia: & $19(32.2 \%)$ \\
\hline ALL & $36(61.0 \%)$ \\
\hline AML & $4(6.8 \%)$ \\
\hline OSknown median (25-75\%) [months]: & $16.06(6.9-103.7)$ \\
\hline ALL & $9.29(0.8-28.0)$ \\
\hline AML & $10.85(6.35-11.25)$ \\
\hline Unknown
\end{tabular}

MGMT methylation:

ALL - positive (\%) 8/19 (42.1\%)

AML - positive (\%) $\quad 11 / 36(30.6 \%)$

Unknown - positive (\%) 2/4 (50\%)

NA (\% of all) $0(0 \%)$

p15 methylation:

ALL - positive (\%) $\quad 1 / 17(5.9 \%)$

AML - positive (\%) 11/33 (33.3\%)

Unknown - positive (\%) 2/2 (100\%)

NA (\% of all) 7 (11.9\%)

DNMT3A mutation:

ALL - positive (\%) 0/19 (0\%)

$\mathrm{AML}$ - positive (\%) 3/34 (8.8\%)

Unknown - positive (\%) 0/3 (0\%)

NA (\% of all) $3(5.1 \%)$

TP53 mutation:

ALL - positive (\%) 0/19 (0\%)

AML - positive (\%) 6/36 (16.7\%)

Unknown - positive (\%) 0/4 (0\%)

NA (\% of all) $0(0 \%)$

Global DNA hypermethylation:

ALL - positive (\%) 4/12 (33.3\%)

AML - positive (\%) 6/23 (26.1\%)

Unknown - positive (\%) 1/3 (33.3\%)

NA (\% of all) $21(35.6 \%)$

Global DNA hypomethylation:

\begin{tabular}{lc|}
\hline ALL - positive (\%) & $8 / 12(66.7 \%)$ \\
\hline AML - positive (\%) & $17 / 23(73.9 \%)$ \\
\hline Unknown - positive (\%) & $2 / 3(66.7 \%)$ \\
\hline NA (\% of all) & $21(35.6 \%)$
\end{tabular}

$N A-$ not assessed. 
(COBRA). Combined bisulfite restriction analysis was estimated using the MethylEdge Bisulfite Conversion System (Promega) according to the manufacturer's instructions. The $p 15$ gene was amplified by PCR using TaKaRa EpiTaq HS polymerase for bisulfite treated DNA (Takara Bio Inc.) with the following primers: forward: 5'-GGAGTTTAAGGGGGTGGG-3' and reverse: 5'-CCTAAATTACTTCTAAAAAAAAAC-3' (Institute of Biochemistry and Biophysics Polish Academy of Sciences, PL). The amplification was carried in a $20 \mu \mathrm{l}$ reaction mixture containing $1 \mu \mathrm{l}$ of template DNA, $0.7 \mu \mathrm{l}$ of $10 \mathrm{mM}$ each primer, $0.6 \mu \mathrm{l} 10 \mathrm{mM}$ dNTPs, $1.2 \mu \mathrm{l}$ of $10 \mathrm{mM}$ $\mathrm{MgCl}_{2}$, and $0.1 \mu \mathrm{l}$ of $5 \mathrm{U} / \mu \mathrm{l}$ EpiTaq HS polymerase with $2 \mu \mathrm{l}$ of 10X EpiTaq PCR Buffer. The PCR conditions were as follows: initial activation of the EpiTaq HS polymerase for $15 \mathrm{~min}$ at $95^{\circ} \mathrm{C}$, followed by 35 cycles of $1 \mathrm{~min}$ denaturation at $94^{\circ} \mathrm{C}$, annealing for $1 \mathrm{~min}$ at $53^{\circ} \mathrm{C}$ and extension for $1 \mathrm{~min}$ at $72^{\circ} \mathrm{C}$ and a final $10 \mathrm{~min}$ extension step at $72^{\circ} \mathrm{C}$. After amplification, PCR products were digested with a specific BstUI restriction enzyme (ABO) that digests alleles that were methylated prior to bisulfite treatment. After digestion, DNA fragments were separated in a $2.5 \%$ agarose gel stained with Midori Green DNA Stain (Nippon Genetics). Product size of the unmethylated DNA was $193 \mathrm{bp}$ and the methylated DNA fragment was $115 \mathrm{bp}$.

\section{Global DNA methylation}

Global DNA methylation levels of 40 cases were measured using the 5-mC DNA ELISA Kit (Zymo Research Corporation, Irvine, CA., USA) according to the manufacturer's protocol. In brief, 100 ng of unknown 5-mC DNA samples was used for analysis. Anti-5-methylcytosine monoclonal antibody (Anti-5-mC mAb) that binds specifically to methylated sites of the genome and the HRP-conjugated secondary antibody were used to detect 5-mC. Absorbance at 405-450 nm was measured using an ELISA plate reader (Tecan Infinite 200 PRO). The presence or absence of $5-\mathrm{mC}$ was determined by comparing the absorbance of samples to negative (0\% methylation) and positive $(100 \%$ methylation) controls. A standard curve was generated by preparing 7 mixtures of the negative and positive controls $(0,5,10,25,50,75$ and $100 \%)$. These were assayed in parallel with the samples. All samples were prepared in duplicate. To determine the $5-\mathrm{mC}$ percentage for unknowns DNA samples the following equation, derived from the logarithmic second-order regression, was used: $\% 5-5 \mathrm{mC}=\mathrm{e}^{[(\text {absorbance }-\mathrm{y} \text {-intercept } / / \text { slope }]}$.

\section{Statistical analysis}

Statistical analysis was performed using Statistica 10.0 PL software (StatSoft, Poland). The distribution of variables was tested with the Shapiro-Wilk test and Kolmogorov-Smirnov test with Lilliefors' correction. Because of non-Gaussian distribution of all analyzed variables we used a non-parametric test and the results are shown as the median followed by the interquartile range (IQR). $P<0.05$ was considered as significant and we used the following tests: the Mann-Whitney $U$ test to verify differences between two groups; Pearson's $\chi^{2}$ test and Yates's correction to verify differences in the distribution of categorical variables between groups; and the Mantel-Cox test and Kaplan-Meier curves for analysis of survival.

\section{Results}

\section{Analysis of MGMT methylation status}

We found that in patients with acute leukemia $35.59 \%$ of cases had a methylated MGMT promoter. Methylation of the MGMT promoter was detected in $42.11 \%$ of ALL samples and $30.56 \%$ of AML samples. There were no significant differences in MGMT methylation status between AML and ALL patients. We also found that in the total group of patients $(p=0.03)$ and ALL cases ( $p=$ $0.04)$, as well as AML patients $(p=0.04)$, methylation of MGMT was associated with age at the time of diagnosis (Figure 1). An example of the result of MGMT methylation status is shown in Figure 2.

\section{Analysis of p15 methylation status}

Methylation of the $p 15$ promoter was detected in $23.08 \%$ of all patients, $5.88 \%$ of ALL samples and $33.33 \%$ of AML samples. The difference between myeloid and lymphoblastic leukemia was significant $\left(p=0.02, \chi^{2}=5.5\right)$. There was no association between $p 15$ methylation status and age of diagnosis in any considered group of patients. An example of the result of $p 15$ methylation analysis is shown in Figure 2.

\section{Analysis of global DNA methylation, DNMT3A and TP53 mutation}

We did not find any significant correlation between global DNA methylation, DNMT3A or TP53 mutation and age of diagnosis. Mutation of TP53 was significantly ( $\left.p=0.02, \chi^{2}=5.47\right)$ more frequent in $A M L$ patients than in $\operatorname{ALL}(16.67 \%$ and $0.00 \%$ respectively). DNMT3A mutation was observed in 3 of all 56 (5.36\%) patients, but only in AML samples (8.8\%). There were no significant associations between DNA methylation, DNMT3A gene mutation and type of leukemia. Figure 3 shows an example of the results of DNMT3A (A) and TP53 (B) mutation analysis. 
A

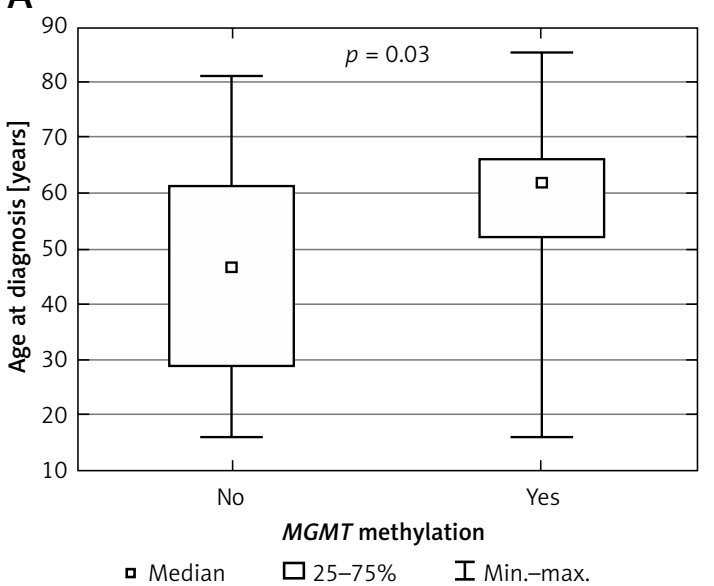

C

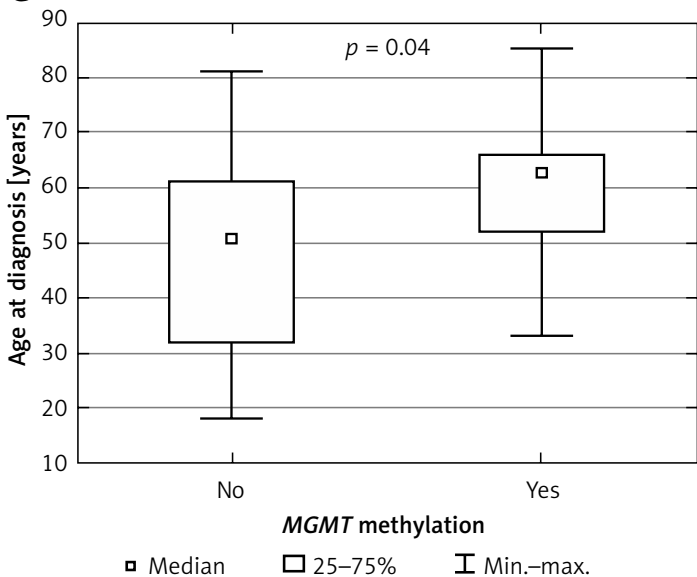

\section{Analysis of relations between examined} molecular parameters

We found that patients with acute leukemia and a methylated promoter for $p 15$ significantly $\left(p=0.04, \chi^{2}=4.25\right)$ more frequently had a mutated TP53 gene (25.00\% vs. 5.00\%). We also found that AML patients with a methylated promoter for $p 15$ significantly $\left(p=0.009, \chi^{2}=6.76\right)$ more frequently had global hypomethylation of DNA detected (100\% vs. $61.54 \%)$. In the group of ALL patients we noted an opposite trend: only patients negative for methylation of the $p 15$ promoter were characterized by global DNA hypomethylation, but this difference did not reach the level of significance. These differences are shown in Figure 4 . We also did not find any differences between female and male patients $(p>0.05)$.

\section{Analysis of survival}

The median overall survival in the group of patients with acute leukemia was 9.9 (16.45) months; when we consider type of leukemia it was 9.29 (27.23) months in AML and 16.06 (96.8) in ALL patients. We also assessed the influence of

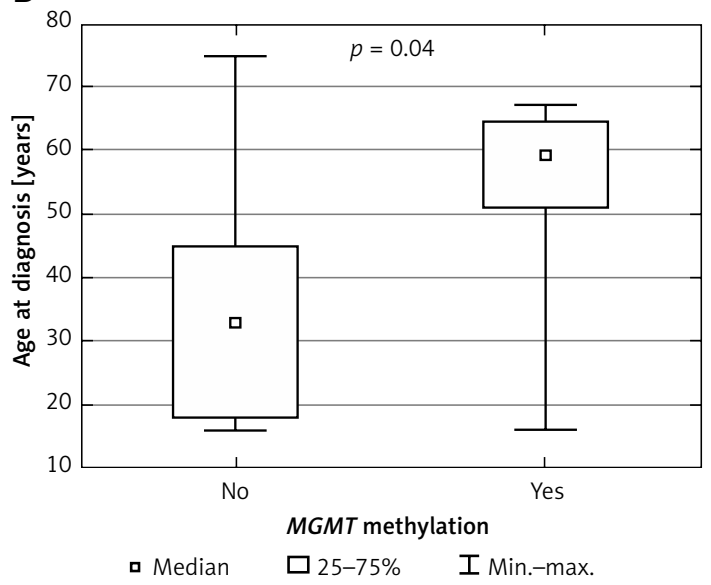

Figure 1. Comparison of age of patients in association with methylation status of MGMT gene in total group of patients (A), ALL patients (B) and AML patients $(C)$

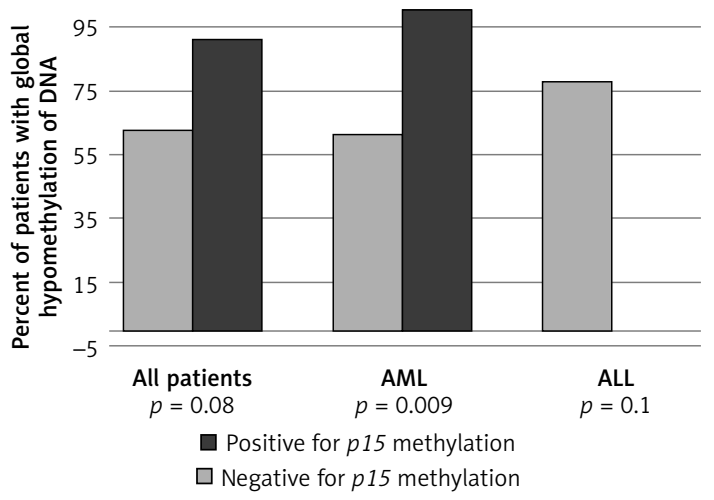

Figure 2. Differences in global DNA hypomethylation in association with type of acute leukemia and methylation of $p 15$

examined molecular parameters on OS. We did not find any correlation between examined parameters and OS of patients.

\section{Discussion}

Genetic disorders are the basis of the malignant transformation process in acute leukemia resulting in abnormal gene expression and cellular transduction pathways and interfering with the 
A

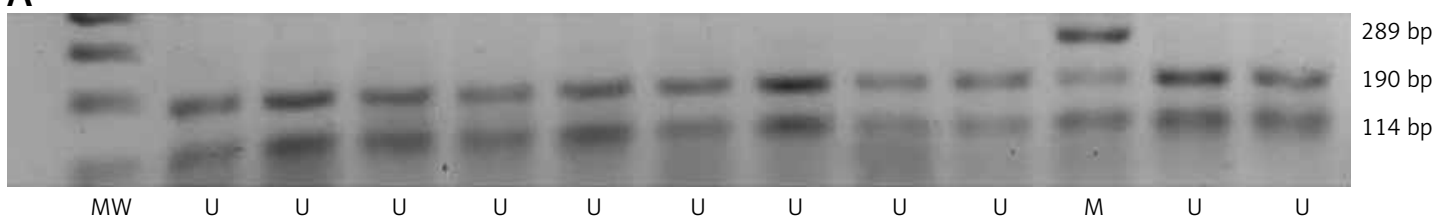

B

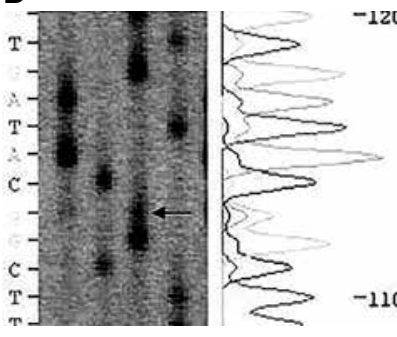

DNA sample with mutation

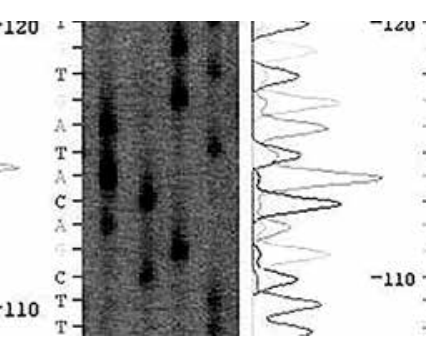

Negative control

Figure 3. DNMT3A and TP53 mutation in acute leukemia patients. A - PCR-RFLP results for the DNMT3A mutation. Presence of 289 bp PCR product indicates that the acute leukemia sample is positive for DNMT3A mutation. $B$ - Example of the result of direct sequencing of a fragment of exon 6 of the TP53 gene

$M-P C R$ product containing mutation, $U-P C R$ product without mutation, MW - molecular weight marker (a 100-bp marker ladder).

A

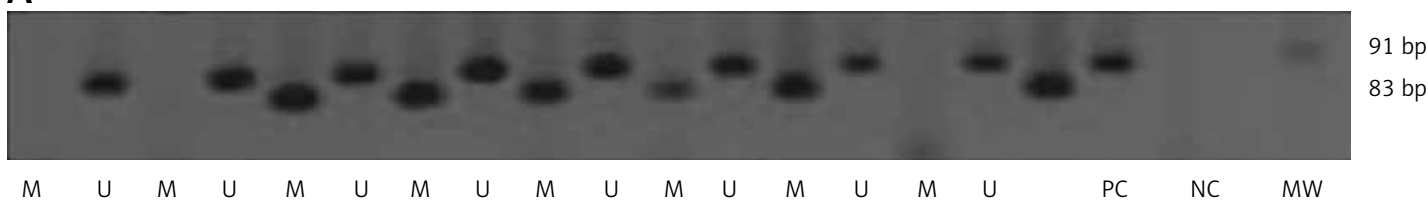

B

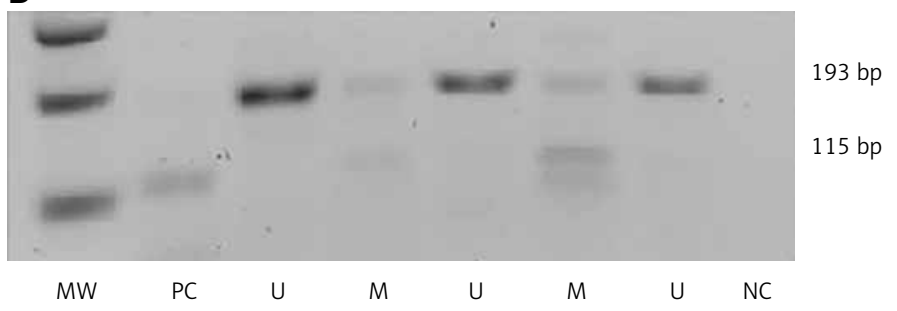

Figure 4. MGMT and $p 15$ methylation in acute leukemia patients. A - MSP results for the MGMT promoter methylation. Presence of 83-bp PCR product indicates that the acute leukemia sample is positive for MGMT methylation. B - COBRA results for $p 15$ methylation. Presence of 115 -bp PCR product indicates that the acute leukemia sample is positive for $p 15$ methylation

$M$ - methylated $P C R$ product, $U$ - unmethylated $P C R$ product, $N C$ - negative control, $P C$ - positive control, $M W$ - molecular weight marker (a 100-bp marker ladder).

proper course of hematopoiesis such as self-renewal, proliferation and differentiation [22]. In the present study we examined the TP53 gene mutations that contribute to the pathogenesis of acute leukemia [23]. TP53 is the most frequently studied gene in cancer and is well characterized in other hematological malignancies, including acute myeloid leukemia (AML) and chronic lymphocytic leukemia (CLL), but data on frequency and the prognostic impact of TP53 mutations in ALL are limited. In our study, TP53 mutation was not found in ALL patients. In ALL, TP53 mutations are rather infrequent. In childhood B-ALL and pediatric T-ALL the reported prevalence is $2-5 \%$ of cases $[24,25]$, whilst in adult patients it is $6.4-11.1 \%$ of cases [26]. On the other hand, TP53 mutations occur in $15.7 \%$ of ALL cases, which increases with age. Moreover, it is associated with MYC rearrangement, low hypodiploidy and a poor prognosis [27]. Other studies have shown that TP53 mutations are frequently detected in $\mathrm{AML}$ with complex karyotype (AML-CK) (59-78\%) [28-30] or therapyrelated $A M L$ [31] but in patients without CK $(2.1 \%)$ [29] and with 17p chromosomal abnormality (2.8\%) [32] it occurs rather rarely. In our study, TP53 mutation was observed in $16.67 \%$ of $\mathrm{AML}$ cases, which is comparable to the frequency reported by others researchers. Shih et al. reported 
that TP53 mutation occurs in $21 \%$ of MDS or AML patients. Moreover, patients with a TP53 mutation or loss of the TP53 locus had worse overall survival compared to those with wild-type TP53 [33]. Our study shows that TP53 mutation occurs in a significant proportion of AML cases. However, we did not find any prognostic value for this alteration, in contrast with other studies [8, 30].

In this study we also examined the DNMT3A mutation. DNMT3A appears to play an important role as a novel prognostic marker in adult AML [34]. Our data demonstrated that the DNMT3A mutation occurs in a small number of cases and only AML patients (8.8\%). In other studies, DNMT3A mutations have been recently found in about $20 \%$ to $41 \%$ of $A M L$ patients [11,35-37]. In contrast, DNMT3A mutations occur at a low frequency in myelodysplastic syndrome patients (8\%) [38] and are also rare in pediatric and childhood AML [39, 40]. Ley et al. observed an adverse prognostic impact of the mutation for AML patients [36], and this finding is consistent with other studies [11, 34, 39]. DNMT3A mutation was significantly associated with advanced age and shorter OS [34, 36]. In our study we found that no mutation was detected in ALL samples. Yan et al. also reported that no mutations were detected in acute myeloid leukemia subtypes M1 to M3 or in acute lymphocytic leukemia [11]. However, in the study by Liu et al., DNMT3A mutations were detected in 3 of all 57 ALL patients [41].

It is now recognized that not only genetic but also epigenetic alterations are important in initiation and progression of both AML and ALL [42, 43]. In the present study, we examined the methylation status of MGMT and $p 15$ genes in AML and ALL patients. Reports in the literature indicate that both genes are unmethylated in normal hematopoietic cells [44-46]. Aberrant p15 methylation has been frequently found in leukemia cell lines, childhood and adult AML and ALL [45, 47-49]. In our study, methylation of the $p 15$ promoter was observed in $23.08 \%$ of all patients but significantly more frequently in AML (33.3\%) than ALL (5.88\%). These findings are consistent with other studies. Hypermethylation of the promoter region of the $p 15$ gene has been detected in primary acute leukemias, with the highest frequency in $A M L$, and it conferred an adverse prognosis [47, 50, 51]. However, we did not find any prognostic value of $p 15$ methylation in acute leukemias. We found that patients with acute leukemia and a methylated promoter for $p 15$ significantly more frequently had a mutated TP53 gene. We also found that $\mathrm{AML}$ patients with a methylated promoter for p15 significantly more frequently had global hypomethylation of DNA detected. Interestingly, in ALL patients we noted the opposite trend: only pa- tients negative for methylation of the $p 15$ promoter were characterized by global DNA hypomethylation, but this difference was not statistically significant. Global hypomethylation is implicated in the development of cancer through promoting genome instability and activation or overexpression of proto-oncogenes [52]. It has been shown that promoter-specific DNA hypermethylation and global DNA hypomethylation are independently associated with the clinical outcome in both ALL and $A M L$ patients $[24,25]$.

MGMT is a DNA-repairing enzyme that removes methyl groups as well as larger adducts at the $\mathrm{O}(6)$ position of guanine [53]. Epigenetic silencing of MGMT expression is frequently observed in several types of cancer as a consequence of transcriptional silencing induced by hypermethylation of the CpG island of the promoter of the MGMT gene [54-56]. Recent studies also indicate an association between the methylation status of the MGMT promoter and the development of MDS [57], adult AML [58, 59] and childhood ALL $[60,61]$. In our study we found methylation of the MGMT promoter in $35.59 \%$ of patients with acute leukemia, but there was no significant difference in MGMT methylation status between AML and ALL patients. Other studies have indicated that MGMT methylation occurs rather rarely (8-11\%) $[60,61]$. However, chemotherapy-induced MGMT methylation status differed among various $A M L$ subtypes, gender and age of patients. Changes in MGMT methylation status were more frequent among M4 subtype patients (50\%) and were not detected in M3 or M5 subtypes [58]. In several studies, methylation of the MGMT gene promoter has been associated with improved prognosis in young [62] and elderly [63] patients with newly diagnosed glioblastoma and in B-DLBCL patients [64]. However, no significant correlation has been found between OS and MGMT promoter methylation status in colorectal adenocarcinoma patients [56]. Our findings showed that methylation of the MGMT gene promoter was significantly associated with the age of diagnosis and there was no correlation of methylation of the MGMT gene promoter as an independent prognostic factor and OS of patients. Interestingly, Kraguljac Kurtovic et al. showed that concomitant aberrant hypermethylation status of $p 15$ and MGMT genes in AML allows stratification of patients with AML into potentially distinct groups with different prognosis [59], but this relationship was not confirmed in our study. Moreover, Lenz et al. demonstrated that MGMT methylation does not seem to be involved in the pathogenesis of AML, because it was not detectable in the examined AML patient samples [19].

In conclusion, genetic and epigenetic abnormalities play a significant role in the pathogene- 
sis of acute leukemia as well as being important prognostic and predictive factors. MGMT promoter methylation was found to be associated with age at diagnosis in both AML and ALL patients. It can be considered as a biomarker for risk group stratification and prognosis in acute leukemias. However, a limitation of our study is the small study group size. Therefore, further research in a larger group of patients is required to assess the clinical utility of MGMT methylation.

\section{Acknowledgments}

Dominika Sobieszkoda and Joanna Czech equal contribution.

The study was conducted at the University of Rzeszow and the Medical University of Lodz.

This study was supported by a grant of the National Science Centre, Poland, no. 2011/01/B/ NZ4/03345. The study was performed within the project 'Centre for Innovative Research in Medical and Natural Sciences' carried out by the University of Rzeszow, co-financed within the Regional Operational Programme for the Podkarpackie Province for the years 2007-2013, contract number UDA-RPPK.01.03.00-18-004/12-00.

\section{Conflict of interest}

The authors declare no conflict of interest.

\section{References}

1. Zuckerman T, Rowe JM. Pathogenesis and prognostication in acute lymphoblastic leukemia. F1000Prime Rep 2014; 6: 59.

2. Estey E, Dohner H. Acute myeloid leukaemia. Lancet 2006; 368: 1894-907.

3. Vardiman JW, Thiele J, Arber DA, et al. The 2008 revision of the World Health Organization (WHO) classification of myeloid neoplasms and acute leukemia: rationale and important changes. Blood 2009; 114: 937-51.

4. Marcucci G, Yan P, Maharry K, et al. Epigenetics meets genetics in acute myeloid leukemia: clinical impact of a novel seven-gene score. J Clin Oncol 2014; 32: 548-56.

5. Figueroa ME, Chen SC, Andersson AK, et al. Integrated genetic and epigenetic analysis of childhood acute lymphoblastic leukemia. J Clin Invest 2013; 123: 3099-111.

6. Hollstein M, Hergenhahn M, Yang Q, et al. New approaches to understanding $\mathrm{p} 53$ gene tumor mutation spectra. Mutat Res 1999; 431: 199-209.

7. Khoury MP, Bourdon JC. The isoforms of the $\mathrm{p} 53$ protein. Cold Spring Harb Perspect Biol 2010; 2: a000927.

8. Nakano Y, Naoe T, Kiyoi H, et al. Prognostic value of p53 gene mutations and the product expression in de novo acute myeloid leukemia. Eur J Haematol 2000; 65: 23-31.

9. Gustafsson B, Axelsson B, Gustafsson B, et al. MDM2 and p53 in childhood acute lymphoblastic leukemia: higher expression in childhood leukemias with poor prognosis compared to long-term survivors. Pediatr Hematol Oncol 2001; 18: 497-508.

10. Li Y, Zhu B. Acute myeloid leukemia with DNMT3A mutations. Leuk Lymphoma 2014; 55: 2002-12.
11. Yan XJ, Xu J, Gu ZH, et al. Exome sequencing identifies somatic mutations of DNA methyltransferase gene DNMT3A in acute monocytic leukemia. Nat Genet 2011; 43: 309-15.

12. Fathi AT, Abdel-Wahab O. Mutations in epigenetic modifiers in myeloid malignancies and the prospect of novel epigenetic-targeted therapy. Adv Hematol 2012; 2012: 469592.

13. Oki Y, Issa JP. Epigenetic mechanisms in $\mathrm{AML}$ - a target for therapy. Cancer Treat Res 2010; 145: 19-40.

14. Das PM, Singal R. DNA methylation and cancer. J Clin Oncol 2004; 22: 4632-42.

15. Garcia-Manero G, Bueso-Ramos C, Daniel J, et al. DNA methylation patterns at relapse in adult acute lymphocytic leukemia. Clin Cancer Res 2002; 8: 1897-903.

16. Kroeger H, Jelinek J, Estecio MR, et al. Aberrant CpG island methylation in acute myeloid leukemia is accentuated at relapse. Blood 2008; 112: 1366-73.

17. Guo SX, Taki T, Ohnishi H, et al. Hypermethylation of p16 and p15 genes and RB protein expression in acute leukemia. Leuk Res 2000; 24: 39-46.

18. Galm O, Herman JG, Baylin SB. The fundamental role of epigenetics in hematopoietic malignancies. Blood Rev 2006; 20: 1-13.

19. Lenz G, Hutter G, Hiddemann W, et al. Promoter methylation and expression of DNA repair genes $\mathrm{hMLH} 1$ and MGMT in acute myeloid leukemia. Ann Hematol 2004; 83: 628-33.

20. Wojcik I, Szybka M, Golanska E, et al. Abnormalities of the P53, MDM2, BCL2 and BAX genes in acute leukemias. Neoplasma 2005; 52: 318-24.

21. Wang $Y, Y u Y, Y e R$, et al. An epigenetic biomarker combination of PCDH17 and POU4F2 detects bladder cancer accurately by methylation analyses of urine sediment DNA in Han Chinese. Oncotarget 2016; 7: 2754-64.

22. Kumar CC. Genetic abnormalities and challenges in the treatment of acute myeloid leukemia. Genes Cancer 2011; 2: 95-107.

23. Zhao Z, Zuber J, Diaz-Flores E, et al. p53 loss promotes acute myeloid leukemia by enabling aberrant self-renewal. Genes Dev 2010; 24: 1389-402.

24. Deneberg S, Grovdal M, Karimi M, et al. Gene-specific and global methylation patterns predict outcome in patients with acute myeloid leukemia. Leukemia 2010; 24: 932-41.

25. Roman-Gomez J, Jimenez-Velasco A, Agirre X, et al. Promoter hypermethylation and global hypomethylation are independent epigenetic events in lymphoid leukemogenesis with opposing effects on clinical outcome. Leukemia 2006; 20: 1445-8.

26. Chen F, Huang T, Ren $Y$, et al. Clinical significance of $\mathrm{CDH} 13$ promoter methylation as a biomarker for bladder cancer: a meta-analysis. BMC Urol 2016; 16: 52.

27. Kim YJ, Kim WJ. Can we use methylation markers as diagnostic and prognostic indicators for bladder cancer? Investig Clin Urol 2016; 57 Suppl. 1: S77-88.

28. Rucker FG, Schlenk RF, Bullinger L, et al. TP53 alterations in acute myeloid leukemia with complex karyotype correlate with specific copy number alterations, monosomal karyotype, and dismal outcome. Blood 2012; 119: 2114-21.

29. Wang K, Tian Y, Xu H. Improved noninvasive bladder cancer diagnosis using urine sediments and novel DNA methylation biomarker panels. Clin Lab 2016; 62: 327-36.

30. Bowen D, Groves MJ, Burnett AK, et al. TP53 gene mutation is frequent in patients with acute myeloid leuke- 
mia and complex karyotype, and is associated with very poor prognosis. Leukemia 2009; 23: 203-6.

31. Pedersen-Bjergaard J, Christiansen DH, Desta F, et al. Alternative genetic pathways and cooperating genetic abnormalities in the pathogenesis of therapy-related myelodysplasia and acute myeloid leukemia. Leukemia 2006; 20: 1943-9.

32. Qi D, Li J, Jiang M, et al. The relationship between promoter methylation of $\mathrm{p} 16$ gene and bladder cancer risk: a meta-analysis. Int J Clin Exp Med 2015; 8: 20701-11.

33. Shih AH, Chung SS, Dolezal EK, et al. Mutational analy sis of therapy-related myelodysplastic syndromes and acute myelogenous leukemia. Haematologica 2013; 98 908-12.

34. Thol F, Damm F, Ludeking A, et al. Incidence and prognostic influence of DNMT3A mutations in acute myeloid leukemia. J Clin Oncol 2011; 29: 2889-96.

35. Yamashita Y, Yuan J, Suetake I, et al. Array-based genomic resequencing of human leukemia. Oncogene 2010; 29: 3723-31.

36. Ley TJ, Ding L, Walter MJ, et al. DNMT3A mutations in acute myeloid leukemia. N Engl J Med 2010; 363 2424-33.

37. Westman MK, Pedersen-Bjergaard J, Andersen MT, et al. IDH1 and IDH2 mutations in therapy-related myelodysplastic syndrome and acute myeloid leukemia are associated with a normal karyotype and with $\operatorname{der}(1 ; 7)$ (q10;p10). Leukemia 2013; 27: 957-9.

38. Walter MJ, Ding L, Shen D, et al. Recurrent DNMT3A mutations in patients with myelodysplastic syndromes. Leukemia 2011; 25: 1153-8.

39. Thol F, Heuser M, Damm F, et al. DNMT3A mutations are rare in childhood acute myeloid leukemia. Haematologica 2011; 96: 1238-40.

40. Hollink IH, Feng Q, Danen-van Oorschot AA, et al. Low frequency of DNMT3A mutations in pediatric $A M L$, and the identification of the $\mathrm{OCl}-\mathrm{AML} 3$ cell line as an in vitro model. Leukemia 2012; 26: 371-3.

41. Liu YN, Zhang N, Wu Y, et al. DNMT3A mutation analy sis in adult patients with acute lymphoblastic leukemia. J Huazhong Univ Sci Technolog Med Sci 2015; 35: 337-42.

42. Burke MJ, Bhatla T. Epigenetic modifications in pediatric acute lymphoblastic leukemia. Front Pediatr 2014; 2: 42.

43. Plass C, Oakes C, Blum W, et al. Epigenetics in acute myeloid leukemia. Semin Oncol 2008; 35: 378-87.

44. Margison GP, Povey AC, Kaina B, et al. Variability and regulation of O6-alkylguanine-DNA alkyltransferase. Carcinogenesis 2003; 24: 625-35.

45. Herman JG, Civin Cl, Issa JP, et al. Distinct patterns of inactivation of p15INK4B and p16INK4A characterize the major types of hematological malignancies. Cancer Res 1997; 57: 837-41.

46. Cameron EE, Baylin SB, Herman JG. p15(INK4B) CpG island methylation in primary acute leukemia is heterogeneous and suggests density as a critical factor for transcriptional silencing. Blood 1999; 94: 2445-51.

47. Herman JG, Jen J, Merlo A, et al. Hypermethylation-associated inactivation indicates a tumor suppressor role for p15INK4B. Cancer Res 1996; 56: 722-7.

48. Batova A, Diccianni MB, Yu JC, et al. Frequent and selective methylation of p15 and deletion of both p15 and p16 in T-cell acute lymphoblastic leukemia. Cancer Res 1997; 57: 832-6.

49. Iravani M, Dhat R, Price CM. Methylation of the multi tumor suppressor gene-2 (MTS2, CDKN1, p15INK4B) in childhood acute lymphoblastic leukemia. Oncogene 1997; 15: 2609-14.
50. Galm O, Wilop S, Luders C, et al. Clinical implications of aberrant DNA methylation patterns in acute myelogenous leukemia. Ann Hematol 2005; 84 Suppl 1: 39-46.

51. Shimamoto T, Ohyashiki JH, Ohyashiki K. Methylation of p15(INK4b) and E-cadherin genes is independently correlated with poor prognosis in acute myeloid leukemia. Leuk Res 2005; 29: 653-9.

52. Laird PW, Jaenisch R. The role of DNA methylation in cancer genetic and epigenetics. Annu Rev Genet 1996; 30: 441-64.

53. Lin YL, Gui SL, Ma JG. Aberrant methylation of $\mathrm{CDH} 11$ predicts a poor outcome for patients with bladder cancer. Oncol Lett 2015; 10: 647-52.

54. Liu Y, Lan Q, Siegfried JM, et al. Aberrant promoter methylation of p16 and MGMT genes in lung tumors from smoking and never-smoking lung cancer patients. Neoplasia 2006; 8: 46-51.

55. Hibi K, Sakata M, Yokomizo K, et al. Methylation of the MGMT gene is frequently detected in advanced gastric carcinoma. Anticancer Res 2009; 29: 5053-5.

56. Oliver JA, Ortiz R, Melguizo C, et al. Prognostic impact of MGMT promoter methylation and MGMT and CD133 expression in colorectal adenocarcinoma. BMC Cancer 2014; 14: 511.

57. Solomon PR, Munirajan AK, Tsuchida N, et al. Promoter hypermethylation analysis in myelodysplastic syndromes: diagnostic and prognostic implication. Indian J Med Res 2008; 127: 52-7.

58. Hong $\mathrm{Q}$, Chen $\mathrm{X}$, Ye $\mathrm{H}$, et al. Association between the methylation status of the MGMT promoter in bone marrow specimens and chemotherapy outcomes of patients with acute myeloid leukemia. Oncol Lett 2016; 11: 2851-6.

59. Kraguljac Kurtovic N, Krajnovic M, Bogdanovic A, et al. Concomitant aberrant methylation of p15 and MGMT genes in acute myeloid leukemia: association with a particular immunophenotype of blast cells. Med Oncol 2012; 29: 3547-56.

60. Takeuchi S, Matsushita M, Zimmermann M, et al. Clinical significance of aberrant DNA methylation in childhood acute lymphoblastic leukemia. Leuk Res 2011; 35 : 1345-9.

61. Gutierrez MI, Siraj AK, Bhargava M, et al. Concurrent methylation of multiple genes in childhood ALL: correlation with phenotype and molecular subgroup. Leukemia 2003; 17: 1845-50.

62. Hegi ME, Diserens AC, Gorlia T, et al. MGMT gene silencing and benefit from temozolomide in glioblastoma. N Engl J Med 2005; 352: 997-1003.

63. Gerstner ER, Yip S, Wang DL, et al. Mgmt methylation is a prognostic biomarker in elderly patients with newly diagnosed glioblastoma. Neurology 2009; 73: 1509-10.

64. Esteller M, Gaidano G, Goodman SN, et al. Hypermethylation of the DNA repair gene $\mathrm{O}(6)$-methylguanine DNA methyltransferase and survival of patients with diffuse large B-cell lymphoma. J Natl Cancer Inst 2002; 94: 26-32. 